\title{
Does Surgeon Experience Impact the Risk of Complications After Bernese Periacetabular Osteotomy?
}

\author{
Eduardo N. Novais MD, Patrick M. Carry BA, Lauryn A. Kestel BS, \\ Brian Ketterman BS, Christopher M. Brusalis BA, Wudbhav N. Sankar MD
}

Published online: 5 August 2016

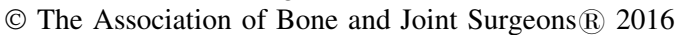

\begin{abstract}
Background Bernese periacetabular osteotomy (PAO) is a technically challenging procedure with potential risk for major complications and a previously reported steep learning curve. However, the impact of contemporary hip preservation fellowships on the learning curve of PAO has not been established.

Questions/purposes (1) What was the frequency of major complications during the PAO learning curve of two surgeons who recently graduated from hip preservation
\end{abstract}

Each author certifies that he or she, or a member of his or her immediate family, has no funding or commercial associations (eg, consultancies, stock ownership, equity interest, patent/licensing arrangements, etc) that might pose a conflict of interest in connection with the submitted article.

All ICMJE Conflict of Interest Forms for authors and Clinical Orthopaedics and Related Research ${ }^{\circledR}$ editors and board members are on file with the publication and can be viewed on request.

Each author certifies that his or her institution approved the human protocol for this investigation, that all investigations were conducted in conformity with ethical principles of research, and that informed consent for participation in the study was obtained.

This work was performed at Children's Hospital Colorado, Aurora, CO, USA, and the Children's Hospital of Philadelphia, Philadelphia, PA, USA.

E. N. Novais $(\bowtie)$

Department of Orthopaedic Surgery, Boston Children's Hospital,

300 Longwood Avenue, Hunnewell Building, Boston,

MA 02215, USA

e-mail: eduardo.novais@childrens.harvard.edu

P. M. Carry, L. A. Kestel, B. Ketterman

Musculoskeletal Research Center, Department of Orthopaedic

Surgery, Children's Hospital Colorado, Aurora, CO, USA

C. M. Brusalis, W. N. Sankar

Division of Orthopaedic Surgery, Children's Hospital of

Philadelphia, Philadelphia, PA, USA fellowships? (2) Is increasing level of experience associated with the risk of a complication and with operative time, a possible surrogate measure of surgical performance?

Methods We retrospectively studied 81 PAOs performed by one of two surgeons who recently graduated from a hip preservation fellowship during their first 4 years of practice in two institutions. One of the surgeons participated as a fellow in 78 PAOs with an increasing level of responsibility during the course of 1 full year. The other surgeon performed 41 PAOs as a fellow during 6 months, also with an increasing level of responsibility during that time. There were $68(84 \%)$ female and $13(16 \%)$ male patients (mean age, 18 years; range, 10-36 years). The frequency of complications was recorded early and at 1 year after surgery and graded according to a validated classification system describing five grades of complications. Complications that required surgical intervention (Grade III) and life-threatening complications (Grade IV) were considered major complications. Persistent pain after surgery, although considered a failure of PAO, was not considered a surgical complication as a result of the multifactorial etiology of pain after hip-preserving surgery. However, early reoperation and revision surgery were counted as complications. To evaluate the association between increasing level of experience and the occurrence of complications, we divided each surgeon's experience into his first 20 procedures (initial interval) and his second 20 (experienced interval) to test whether the incidence of complications or operative time was different between the two intervals. Because the association between experience and the likelihood of a complication was estimated to be consistent between the two surgeons, the analysis was performed with data pooled from the two surgeons. To test whether there was a difference in the likelihood of a complication in the 
initial and the experienced intervals, a multivariate logistic regression analysis was performed and the adjusted risk of a complication between the two intervals was calculated. Linear regression analyses were used to test the association between surgeon level of experience and operative time.

Results The overall incidence of major (Grade III or Grade IV) complications was 6\% (95\% confidence interval [CI], 2\%-14\%). These included deep infection (3\% [three of 81 ]), intraoperative posterior column fracture (1\% [one of 81]), and pulmonary embolism (1\% [one of 81 ]). With the numbers available, the risk of a complication did not decrease with increasing surgeon experience. After controlling for body mass index and surgeon, the frequency of a complication did not decrease in the experienced interval relative to the initial interval (odds ratio, 0.78 ; 95\% CI, $0.25-2.4 ; \mathrm{p}=0.6623)$. The adjusted risk difference between the experienced interval relative and the initial interval was $6 \%(95 \% \mathrm{CI},-11 \%$ to $23 \%)$. When experience was modeled as a continuous variable (number of PAOs performed), increasing experience was not associated with a lower likelihood of a complication (odds ratio per one PAO increase in experience, 0.99 ; 95\% CI, 0.94 $1.04 ; \mathrm{p}=0.5478$ ). However, after adjusting for body mass index and surgeon, increased experience was associated with a reduction in operative time (slope [change in log operative time per one procedure increase in experience], $-0.005 ; 95 \% \mathrm{CI},-0.009$ to $-0.0005 ; \mathrm{p}=0.0292$ ). For every one additional PAO increase in experience, there was a $0.45 \%$ decrease in operative time $(95 \%$ CI, $0.05 \%-0.86 \%$ decrease].

Conclusions With a case exposure greater than 40 PAOs and progressive surgical responsibility during contemporary structured training, two young surgeons were able to perform PAO with a low risk of complications. However, even with that surgical experience before independent practice, surgical time decreased over the first 40 PAOs they performed independently. Our data may help guide orthopaedic residency and hip preservation fellowship programs in establishing training requirements and assessing competency in PAO.

Level of Evidence Level III, therapeutic study.

\section{Introduction}

Since its description in 1988 [10], the Bernese periacetabular osteotomy (PAO) has become one of the most accepted surgical treatments for symptomatic hip dysplasia [5, 24]. The Bernese PAO, however, is a technically challenging procedure with a historical proportion of major complications reported between $4 \%$ and $46 \%$ [3, 6-8, 12, 24-28]. Although few patient risk factors [16-18, 21, 25] have been described, the surgeon's experience has been reported as the leading factor associated with PAO complications [6]. Several studies have shown an inverse relationship between complication risk and surgeon experience $[8,10,12,20$, 26, 27]. One study [12] reviewed over 508 osteotomies performed by the originator of the technique and found that $85 \%$ of the technical complications occurred during the first 50 procedures, suggesting an important learning curve associated with PAO.

Nevertheless, contemporary studies have described PAO as a safe procedure with a low incidence of major complications when performed by experienced surgeons $[25,28]$. A recent study reported a $6 \%$ risk of major complications in 205 patients undergoing PAO by a group of 10 surgeons with an average of 9 years (range, 1-37 years) of experience [28]. However, there is little evidence about length and type of training necessary to achieve this level of expertise.

Therefore, in this study we asked: (1) What was the frequency of major complications during the PAO learning curve of two surgeons who recently graduated from hip preservation fellowships? (2) Is increasing level of experience associated with the risk of a complication and with operative time, a possible surrogate measure of surgical performance?

\section{Materials and Methods}

After obtaining institutional review board approval from two centers, a retrospective study was designed to collect data from all patients who underwent PAO between October 2009 and December 2014. This study includes the first consecutive osteotomies performed by each of two surgeons (ENN, WNS) during their first 4 years of independent practice. The study consisted of 81 patients (mean age, 18 years; range, 10-36 years), 43 (53\%) from Surgeon 1 and 38 (47\%) from Surgeon 2. Surgeon 1 participated as a fellow in 78 PAOs with increasing level of responsibility during the course of 1 full year. Surgeon 2 performed 41 PAOs as a fellow during 6 months, also with an increasing level of responsibility during that time. Both surgeons attended an organized, structured hip preservation fellowship program in an institution that has more than 20 years of experience in PAO. The fellowship was organized in such a way that the fellows rotated with two orthopaedic surgeons who specialized in hip preservation procedures. Before attending the hip preservation fellowship, both Surgeon 1 and Surgeon 2 completed a year of pediatric fellowship training after orthopaedic surgery residency. In addition, Surgeon 1 had additional surgical experience in a 1-year fellowship in musculoskeletal oncology and Surgeon 2 had additional experience in an infantile hip dysplasia fellowship for a 6-month period. 
Preoperative variables including age, gender, body mass index, and secondary diagnosis were recorded from medical records. The lateral center-edge angle, the acetabular inclination angle, and the anterior center-edge angle were assessed on radiographs. Most hips (98\% [79 of 81]) were affected by developmental dysplasia. Acetabular retroversion leading to femoroacetabular impingement was the diagnosis in two hips (3\%). Secondary diagnoses included osteonecrosis of the femoral head (two of 81 [3\%]), arthrogryposis (two of 81 [3\%]), Down syndrome (two of 81 [3\%]), Charcot-Marie-Tooth disease (one of 81 [1.2\%]), cerebral palsy (one of 81 [1.2\%]), unspecified chromosomal abnormality (one of 81 [1.2\%]), and polymyositis (one of 81 [1.2\%]). Operative records were reviewed for collection of operative time, defined as skin incision to completion of skin closure, and secondary procedures performed in combination with PAO (Table 1).

The PAO was performed according to the technique described by Ganz et al. [10] through a Smith-Petersen modified bikini approach with preservation of the abductor musculature [14]. In 38 of the 81 patients (47\%), the origin of the rectus femoris tendon was preserved [15]. Briefly, the ischium was cut at the infracotyloid groove under C-arm visualization followed by osteotomy of the superior pubic ramus medial to the iliopectineal eminence. Next, with an oscillating saw a supraacetabular osteotomy was performed. The posterior column was then cut midway between the joint and the sciatic notch. Finally the fragment was mobilized, freed, and repositioned. The acetabular fragment was fixed with three to four 3.5-mm or 4.5-mm screws after appropriate correction was confirmed by intraoperative fluoroscopy. All patients received preoperative antibiotic prophylaxis that continued for 24 hours after surgery. Postoperatively, patients were placed on crutches with restricted partial weightbearing for 6 to 8 weeks until early radiographic signs of healing were observed. For the purpose of this study, early complications were recorded during the 6-week postoperative visit for Surgeon 1 and 8 weeks for Surgeon 2. The frequency of subsequent followup visits varied by center, but patients of both surgeons returned at 1 year for a postoperative appointment.

The primary outcome variable of interest was the occurrence of complications during the first year after surgery. Complications that occurred $<10$ weeks after surgery were considered early complications. Complications that occurred between the 10-week and the 1-year followup visits were considered late complications. Complications were graded according to an adaptation of the Clavien-Dindo [4, 9] grading system, which has been validated in hip preservation surgery [23]. According to this system, a Grade I complication does not lead to a change in routine postoperative care and requires no further treatment. A Grade II complication is a deviation from the postoperative course requiring outpatient pharmacologic treatment or close monitoring. Grade III complications require invasive surgical, endoscopic, or radiographic treatment often on an inpatient basis. Grade IV complications are life-threatening events or not treatable and associated with high morbidity and potential for permanent disability. A Grade V complication is death. In this study, a major complication was defined as Grade III or higher. Each complication was analyzed individually; thus, multiple complications could be recorded for one patient. For the purpose of the study, persistent pain after PAO was not considered a surgical complication of the procedure. The

Table 1. Comparison of demographics and clinical characteristics during the initial interval and the experienced interval

\begin{tabular}{lcc}
\hline Demographics and clinical characteristics & $\begin{array}{l}\text { Initial interval } \\
(\mathrm{N}=40)\end{array}$ & $\begin{array}{l}\text { Experienced interval } \\
(\mathrm{N}=41)\end{array}$ \\
\hline Gender, number (\%) & $7(17 \%)$ & $6(15 \%)$ \\
$\quad$ Male & $33(82 \%)$ & $35(85 \%)$ \\
$\quad$ Female & $19(47 \%)$ & $24(58 \%)$ \\
Number of prior procedures, number (\%) & $21(52 \%)$ & $17(41 \%)$ \\
None & & \\
Multiple & $35(87 \%)$ & $34(83 \%)$ \\
Secondary diagnosis, number (\%) & $5(12 \%)$ & $7(17 \%)$ \\
One or more secondary diagnoses & $6( \pm 12)$ & $6( \pm 13)$ \\
No secondary diagnoses & $20( \pm 8)$ & $22( \pm 9)$ \\
Baseline LCEA (degrees), mean (SD) & $5( \pm 21)$ & $4( \pm 14)$ \\
Baseline Tönnis angle (degrees), mean (SD) & $24( \pm 4)$ & $22( \pm 5)$ \\
Baseline ACEA angle (degrees), mean (SD) & $18( \pm 5)$ & $18( \pm 6)$ \\
BMI (kg/m $\left.{ }^{2}\right)$, mean (SD) & 0.320 \\
Age (years), mean (SD) & & 0.562 \\
\hline
\end{tabular}

LCEA = lateral center-edge angle; ACEA = anterior center-edge angle; BMI = body mass index. 
etiology of persistent pain after PAO is multifactorial, and it has therefore typically been reported as treatment failure rather than as a surgical-related complication [28]. Because persistent pain may lead to further operative interventions, early reoperation and revision were counted as complications.

\section{Statistical Analysis}

The incidence of major complications (Grades III and IV) in the cohort was recorded. Exact $95 \%$ confidence intervals were used to express the variability in the incidence estimate. To evaluate the association between increasing level of experience and overall complication risk, we divided each surgeon's experience into his first 20 procedures (initial interval) and his second 20 procedures (experienced interval). To determine whether data from the two surgeons could be pooled together, we tested whether the association between experience (cumulative number of PAOs performed by the treating surgeon before that procedure) and overall complication risk was different across the two surgeons. The association between experience and the likelihood of a complication was consistent across surgeons ( $\mathrm{p}=0.8860$; Fig. 1) and thus we concluded it was appropriate to pool data from the two surgeons. A multivariable logistic regression analysis was used to test the null hypothesis of no difference in the likelihood of a

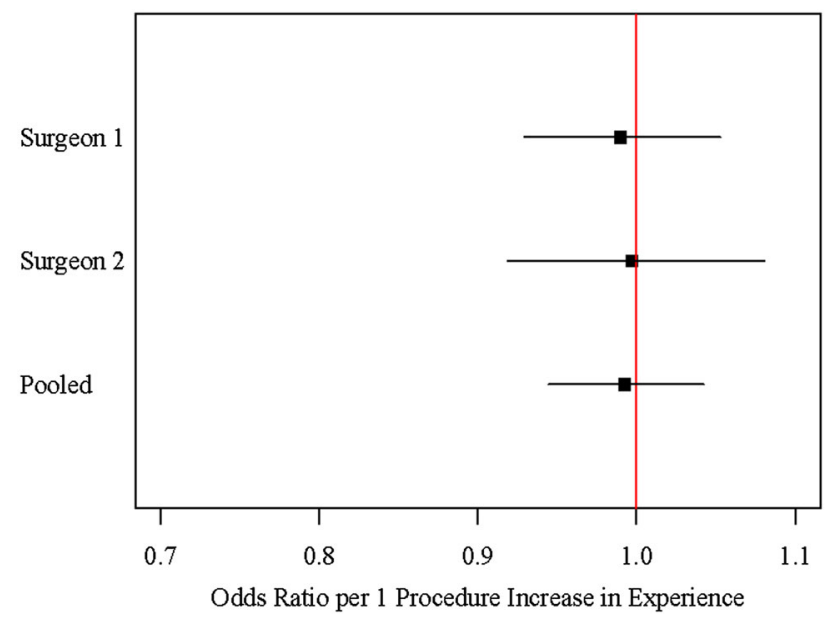

Fig. 1 Association between experience and the likelihood of a complication is described as odds ratio per one PAO increase in experience. To determine whether data from the two surgeons could be pooled together, we tested whether the association between experience (cumulative number of PAOs performed by the treating surgeon before that procedure) and overall complication risk was different across the two surgeons. The association between experience and the likelihood of a complication was consistent across surgeons $(\mathrm{p}=0.8860)$ and thus we concluded it was appropriate to pool data from the two surgeons. complication in the two intervals. To aid the interpretation, the adjusted risk difference between the two intervals was calculated according to the methods described by Austin [2]. A linear regression analysis was used to test the association between surgeon level of experience and operative time. Based on the skewed distribution of operative time, log transformation was applied to this variable. Differences in demographics and clinical characteristics between the initial interval and the experienced interval were used to identify potential confounding variables that were included in the multivariable models (Table 1). Surgeon was also adjusted for in the multivariable models as a potential surrogate measure of institutional-specific factors that were not directly measured in the current study.

\section{Results}

Major (Grade III or Grade IV) complications occurred in $6 \%$ (95\% confidence interval [CI], 2\%-14\%) of all patients. There were no deaths (Grade V). All Grade III and Grade IV complications occurred between 0 and 10 weeks after surgery. These included deep infection (3\% [three of $81])$, intraoperative posterior column fracture $(1.2 \%$ [one of 81$]$ ), and pulmonary embolism (1.2\% [one of 81 ]). The three cases of deep infection may have been related to long surgical procedure time because the median operative time was 320 minutes. The one posterior column fracture in the series occurred intraoperatively on the second strike of the ischial osteotomy when a crack propagated above the ischial spine. The fracture was nondisplaced; however, the PAO was halted and the definitive procedure was performed later during the same hospitalization after further imaging identified a nondisplaced fracture. One large wound dehiscence was managed with irrigation and surgical débridement in combination with negative pressure wound therapy. No major complications ( $0 \%$ [zero of 81 ]) occurred between the 10th postoperative week and 1 year postoperatively (Table 2). At 1 year postoperatively, there was no disability related to the complications that had resolved completely.

With the numbers available, the risk of a complication did not decrease with increasing surgeon experience. After adjusting for body mass index and surgeon, the odds of a complication did not decrease in the experienced interval (last 20 PAOs) relative to the initial interval (first 20 PAOs; odds ratio, $0.78 ; 95 \% \mathrm{CI}, 0.25-2.40 ; \mathrm{p}=0.6623)$. The adjusted risk difference between the experienced interval relative to the initial interval was $5.9 \%(95 \% \mathrm{CI},-11.2 \%$ to $23.1 \%$ ). When experience was modeled as a continuous variable (number of PAOs performed), increased experience was not associated with a decrease in the likelihood of a complication (odds ratio per one PAO increase in 
Table 2. Description, severity level, and management of all complications

\begin{tabular}{|c|c|c|c|}
\hline Description & Number & $\begin{array}{l}\text { Clavien-Dindo } \\
\text { modified grade }\end{array}$ & Management \\
\hline \multicolumn{4}{|l|}{ Early complications $(0-10$ weeks $)$} \\
\hline Pulmonary embolism & 1 & 4 & Anticoagulant medication \\
\hline Deep infection & 2 & 3 & Incision and drainage + antibiotics \\
\hline Intraoperative nondisplaced posterior column fracture & 1 & 3 & $\begin{array}{l}\text { PAO performed as a staged procedure }+ \\
\text { additional weightbearing restrictions }\end{array}$ \\
\hline Wound dehiscence & 1 & 3 & $\begin{array}{l}\text { Débridement }+ \text { negative pressure wound } \\
\text { therapy }\end{array}$ \\
\hline $\begin{array}{l}\text { Neurapraxia of the motor branch of the deep peroneal nerve related to } \\
\text { a pressure injury to the fibular head area by compression stockings }\end{array}$ & 1 & 2 & $\begin{array}{l}\text { Monitoring and followup to the extensor } \\
\text { hallux longus, which recovered } \\
\text { completely }\end{array}$ \\
\hline Peroneal nerve dysesthesia* & 1 & 2 & Pharmacologic treatment (gabapentin) \\
\hline Left inferior pubic ramus stress fracture & 1 & 2 & Crutches for 4 weeks \\
\hline Calf pain & 1 & 1 & $\begin{array}{l}\text { Doppler to assess for DVT, which was } \\
\text { negative }\end{array}$ \\
\hline Wound dehiscence & 1 & 1 & None; secondary wound healing \\
\hline \multicolumn{4}{|l|}{ Late complications (10 weeks to 12 months) } \\
\hline Psoas tenosynovitis/snapping & 1 & 2 & NSAIDs and physical therapy \\
\hline Lateral femoral cutaneous nerve dysesthesia & 1 & 2 & $\begin{array}{l}\text { Pharmacologic treatment }+ \text { lidocaine } \\
\text { patches }\end{array}$ \\
\hline Heterotopic ossification-Brooker Grade II & 7 & 1 & None \\
\hline
\end{tabular}

* Peroneal nerve dysesthesia developed as pain and tingling on the dorsal aspect of the foot of a 13-year-old girl with arthrogryposis and multiple previous surgeries on her foot, including application of an external fixator; this patient had always experienced pain in her foot that was thought to be worsened for the first 2 months after PAO; PAO = periacetabular osteotomy; DVT = deep vein thrombosis; NSAIDs = nonsteroidal antiinflammatory drugs.

experience, $0.99 ; 95 \%$ CI, 0.94-1.04; $\mathrm{p}=0.5478$ ). However, as surgeon experience increased, surgical time decreased. Median operative time was 320 minutes (interquartile range, 252-333 minutes) in the initial interval compared with 228 minutes (interquartile range, 279-366 minutes) in the experienced interval. After adjusting for body mass index and surgeon, there was an inverse association between increased experience and operative time (slope [change in log operative time per one PAO increase in experience], $-0.0045 ; 95 \% \mathrm{CI},-0.0086$ to -0.0005 ; $\mathrm{p}=0.0292$ ). For every one PAO increase in experience, there was a $0.45 \%$ decrease in operative time $(95 \% \mathrm{CI}$, $0.05 \%-0.86 \%$ decrease; Fig. 2).

\section{Discussion}

The Bernese PAO is a technically challenging procedure that has gained popularity for the treatment of acetabular dysplasia. The ideal method of training an orthopaedic surgeon to perform PAO has not been established. Training obtained during orthopaedic residency may not be sufficient [19]; therefore, other learning strategies including visiting high-volume hip centers, attending specialty society courses, and practicing the procedure on cadavers have

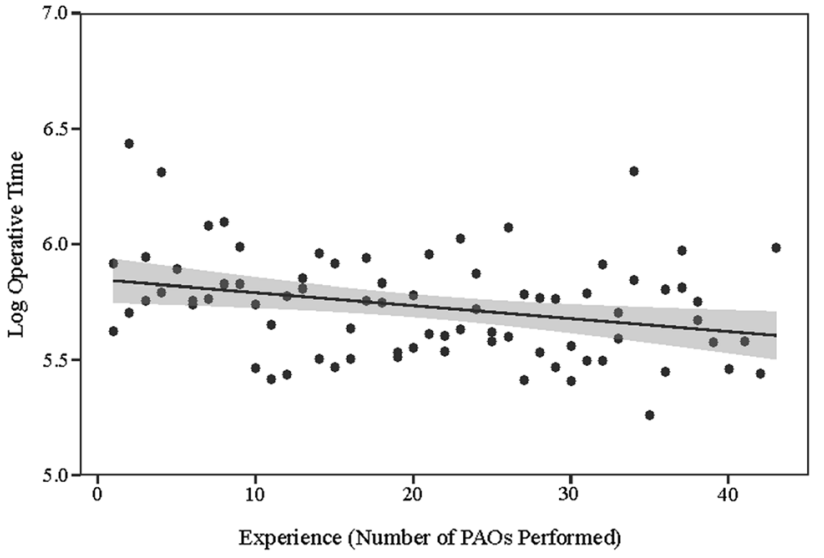

Fig. 2 Operative time during the study period and experience of the surgeon are presented.

been described $[7,8,19,20,25]$. Currently, there are few structured fellowship programs in hip preservation surgery [19]. Previous studies [8, 10, 12, 20, 27] have reported a higher incidence of complications during the first PAOs; however, there are limited data about the learning curve of PAO after contemporary structured training. In this study, we reviewed the PAOs performed by two young surgeons with fellowship training in both pediatric orthopaedic and hip preservation surgery during their first 4 years of 
practice. We found that with a case exposure greater than 40 PAOs and progressive surgical responsibility during training, the two young surgeons were able to perform PAO with a relatively low risk of complications. However, even with that surgical experience before independent practice, surgical time decreased over the first 40 PAOs they performed independently.

This study has several limitations, all of which could cause our results to be best-case estimates of the effect of fellowship training on the learning curve associated with PAO. Indeed, because of these limitations, the learning curve may be longer than our study estimates it to be. First, we did not gather patient-reported outcomes consistently in these patients and so we could not report them here. It is possible that the earlier patients did not do as well as the later ones. Also, in this study, we did not include persistent pain after surgery as a complication, in line with a previous study [28]. However, we did not have any patients undergo revision surgery because of residual pain and failure of PAO in the short term. Second, we do not present the radiographic accuracy of the osteotomy correction. Acetabular repositioning is a key factor that influences the outcome of PAO; however, the clinical effects of poor positioning may not be detected in the short term [1, 13]. It is possible that longer followup of these patients could reveal that surgeon experience influences acetabular positioning and therefore may be a key factor in poor clinical outcomes including residual pain and early degenerative joint disease. We did not report on patient-reported outcomes and radiographic correction because this study was purposely designed to assess surgical complications associated with PAO and not necessarily the efficacy of the procedure.

The retrospective nature of this study design may have also led to an underestimate of the incidence of complications. However, this was a consecutive series including all the patients who underwent surgery during the study period who were consistently seen at an early clinic visit and at least at 1 year after surgery without any loss to followup. Therefore, we believe that all of the major complications affecting patients in this cohort were collected. It is possible, however, that minor complications such as dysesthesia of the lateral femoral cutaneous nerve could be underreported. It is also possible that some complications were influenced by patient-specific factors such as the occurrence of thromboembolism after surgery. However, we believe that by using a validated grading system to classify all the complications, there is consistency in our methodology, which allows comparison of our data to previous studies on this topic $[8,28]$. Fourth, we did not investigate the experience of the surgical team and support nursing staff as a potential risk factor for increased risk of complications. We feel that this would require a prospective controlled study design. Finally, the two surgeons in this study completed a residency program, additional training in a formal pediatric orthopaedics fellowship, and a hip preservation fellowship. Therefore, we caution that the results of our study may not be generalizable to all young orthopaedic surgeons with different training background.

In this study, the frequency of major complications during the first independent 40 PAO cases performed by the two surgeons during their first 4 years in practice was $6 \%$. This is a lower complication rate than previous studies reporting a learning curve associated with PAO surgery. Davey and Santore [8] compared the first 35 PAO cases with the second 35 cases executed by a single surgeon who had prepared for the first PAO by visiting two sites and performed 19 cadaveric procedures during the first year. The surgeon also had extensive experience with other pelvic and femoral procedures. They observed a reduction of moderate and major complications from $46 \%$ to $14 \%$ in the early versus late series. Major complication incidence decreased from $17 \%$ to $3 \%$. Peters et al. [20] reported on the first 73 patients ( 83 hips) who underwent PAO by a single surgeon who had experience with pelvic anatomy and learned the PAO technique by assisting an experienced surgeon, by performing cadaveric surgery four times, and by visiting one of the originators of the operation. Nine of the first 30 hips (30\%) developed a major complication compared with $2 \%$ in the subsequent 53 hips. Trumble et al. [27] reported a $20 \%$ rate of major complications, which occurred early in a series of 123 osteotomies performed using an ilioinguinal approach. Crockarell et al. [7] reported a total of 13 (two peroneal nerve palsies, three fractures of the ischium, three asymptomatic pubic nonunions, and five cases of heterotopic ossification) complications in the first 21 cases for an overall incidence of $60 \%$. Howie et al. [11] published one of the few studies specifically investigating training and the learning curve associated with PAO. During a 12-year study period, the authors reported on 14 patients (15 hips) who underwent PAO through a single approach and described a mentorship program including visits with the originator of the procedure, cadaveric training, and discussion of all the cases with a mentor. They reported two (13\%) major complications (one ischium nonunion and one temporary femoral nerve palsy) in their series.

Contrary to these previous studies, we found a risk of major complications during the first year after PAO similar to incidence estimates associated with experienced surgeons [28]. Zaltz et al. evaluated the complication risk in 205 patients who underwent PAO performed by a group of 10 surgeons who had an average of 9 years of experience with PAO. They reported a $6 \%$ risk of major complications, similar to the rate of complications reported in the current study [28]. In our study, although deep infections 
and one pulmonary embolus did occur, there were no permanent nerve injuries, intraarticular extensions of the osteotomy, loss of fixation, or displaced posterior column fractures from errors in technique. The one posterior column fracture in the series was nondisplaced and occurred on the second strike of the ischial osteotomy when a crack propagated above the ischial spine.

In the present study, the risk of a complication did not decrease with increasing surgeon experience. It is possible that the time spent in postresidency training, including a hip preservation fellowship with graduated levels of responsibility during PAO, allowed the two surgeons to experience a portion of the learning curve under direct supervision. Thawrani et al. reported a similar proportion of complications during their initial 42 PAOs compared with the later 41 PAOs. The surgeons in that study [25] accumulated experience with PAO by scrubbing as first assistant during residency and by visiting with the originator of the technique. However, we did find an inverse relationship between experience (number of PAO procedures performed) and operative time, a possible surrogate measure of surgical performance and learning curve [22]. This is consistent with the study by Davey and Santore [8] that reported a statistically significant reduction in surgical time from 3 hours 48 minutes to 3 hours 24 minutes. Improvement in operative time, however, may not only reflect the surgeon's performance, but may be a result of the entire surgical staff gaining experience with the procedure.

In conclusion, the incidence of major complications for young surgeons with formal structured hip preservation fellowship training was similar during the initial interval (first 20 PAOs) relative to the experienced interval (next 20 PAOs). Although the optimal method of training an orthopaedic surgeon for this complex surgical procedure has not been established, this study suggests that with a case exposure greater than 40 and progressive surgical responsibility during training, two young surgeons were able to perform PAO with a relatively low risk of complications. However, even with that surgical experience before independent practice, surgical time decreased over the first 40 PAOs they performed independently. Our data may help guide orthopaedic residency and hip preservation fellowship programs as they establish training requirements and assess competency in PAO.

\section{References}

1. Albers CE, Steppacher SD, Ganz R, Tannast M, Siebenrock KA. Impingement adversely affects 10-year survivorship after periacetabular osteotomy for DDH. Clin Orthop Relat Res. 2013;471:1602-1614.
2. Austin PC. Absolute risk reductions, relative risks, relative risk reductions, and numbers needed to treat can be obtained from a logistic regression model. J Clin Epidemiol. 2010;63:2-6.

3. Biedermann R, Donnan L, Gabriel A, Wachter R, Krismer M, Behensky H. Complications and patient satisfaction after periacetabular pelvic osteotomy. Int Orthop. 2008;32:611-617.

4. Clavien PA, Barkun J, de Oliveira ML, Vauthey JN, Dindo D, Schulick RD, de Santibanes E, Pekolj J, Slankamenac K, Bassi C, Graf R, Vonlanthen R, Padbury R, Cameron JL, Makuuchi M. The Clavien-Dindo classification of surgical complications: fiveyear experience. Ann Surg. 2009;250:187-196.

5. Clohisy JC, Carlisle JC, Beaule PE, Kim YJ, Trousdale RT, Sierra RJ, Leunig M, Schoenecker PL, Millis MB. A systematic approach to the plain radiographic evaluation of the young adult hip. J Bone Joint Surg Am. 2008;90(Suppl 4):47-66.

6. Clohisy JC, Schutz AL, St John L, Schoenecker PL, Wright RW. Periacetabular osteotomy: a systematic literature review. Clin Orthop Relat Res. 2009;467:2041-2052.

7. Crockarell J Jr, Trousdale RT, Cabanela ME, Berry DJ. Early experience and results with the periacetabular osteotomy. The Mayo Clinic experience. Clin Orthop Relat Res. 1999;363:45-53.

8. Davey JP, Santore RF. Complications of periacetabular osteotomy. Clin Orthop Relat Res. 1999;363:33-37.

9. Dindo D, Demartines N, Clavien PA. Classification of surgical complications: a new proposal with evaluation in a cohort of 6336 patients and results of a survey. Ann Surg. 2004;240:205-213.

10. Ganz R, Klaue K, Vinh TS, Mast JW. A new periacetabular osteotomy for the treatment of hip dysplasias. Technique and preliminary results. Clin Orthop Relat Res. 1988;232:26-36.

11. Howie DW, Beck M, Costi K, Pannach SM, Ganz R. Mentoring in complex surgery: minimising the learning curve complications from peri-acetabular osteotomy. Int Orthop. 2012;36:921-925.

12. Hussell JG, Rodriguez JA, Ganz R. Technical complications of the Bernese periacetabular osteotomy. Clin Orthop Relat Res. 1999;363:81-92.

13. Millis MB, Siebenrock KA, Session P. Report of breakout session: Defining parameters for correcting the acetabulum during a pelvic reorientation osteotomy. Clin Orthop Relat Res. 2012;470:3453-3455.

14. Murphy SB, Millis MB. Periacetabular osteotomy without abductor dissection using direct anterior exposure. Clin Orthop Relat Res. 1999;364:92-98.

15. Novais EN, Kim YJ, Carry PM, Millis MB. The Bernese periacetabular osteotomy: is transection of the rectus femoris tendon essential? Clin Orthop Relat Res. 2014;472:3142-3149.

16. Novais EN, Kim YJ, Carry PM, Millis MB. Periacetabular osteotomy redirects the acetabulum and improves pain in Charcot-Marie-Tooth hip dysplasia with higher complications compared with developmental dysplasia of the hip. J Pediatr Orthop. 2015 Jun 3 [Epub ahead of print].

17. Novais EN, Potter GD, Clohisy JC, Millis MB, Kim YJ, Trousdale RT, Carry PM, Sierra RJ. Obesity is a major risk factor for the development of complications after peri-acetabular osteotomy. Bone Joint J. 2015;97:29-34.

18. Novais EN, Potter GD, Sierra RJ, Kim YJ, Clohisy JC, Schoenecker PL, Trousdale RT, Carry PM, Millis MB. Surgical treatment of adolescent acetabular dysplasia with a periacetabular osteotomy: does obesity increase the risk of complications? $J$ Pediatr Orthop. 2015;35:561-564.

19. Peters CL, Beaule PE, Beck M, Tannast M, Jiranek W, Sierra RJ. Report of breakout session: Strategies to improve hip preservation training. Clin Orthop Relat Res. 2012;470:3467-3469.

20. Peters CL, Erickson JA, Hines JL. Early results of the Bernese periacetabular osteotomy: the learning curve at an academic medical center. J Bone Joint Surg Am. 2006;88:1920-1926. 
21. Polkowski GG, Novais EN, Kim YJ, Millis MB, Schoenecker PL, Clohisy JC. Does previous reconstructive surgery influence functional improvement and deformity correction after periacetabular osteotomy? Clin Orthop Relat Res. 2012;470:516-524.

22. Ramsay CR, Grant AM, Wallace SA, Garthwaite PH, Monk AF, Russell IT. Assessment of the learning curve in health technologies. A systematic review. Int J Technol Assess Health Care. 2000;16:1095-1108.

23. Sink EL, Leunig M, Zaltz I, Gilbert JC, Clohisy J; Academic Network for Conservational Hip Outcomes Research Group. Reliability of a complication classification system for orthopaedic surgery. Clin Orthop Relat Res. 2012;470:2220-2226.

24. Steppacher SD, Tannast M, Ganz R, Siebenrock KA. Mean 20year followup of Bernese periacetabular osteotomy. Clin Orthop Relat Res. 2008;466:1633-1644.
25. Thawrani D, Sucato DJ, Podeszwa DA, DeLaRocha A. Complications associated with the Bernese periacetabular osteotomy for hip dysplasia in adolescents. J Bone Joint Surg Am. 2010; 92:1707-1714.

26. Trousdale RT, Cabanela ME. Lessons learned after more than 250 periacetabular osteotomies. Acta Orthop Scand. 2003; 74:119-126.

27. Trumble SJ, Mayo KA, Mast JW. The periacetabular osteotomy. Minimum 2 year followup in more than 100 hips. Clin Orthop Relat Res. 1999;363:54-63.

28. Zaltz I, Baca G, Kim YJ, Schoenecker P, Trousdale R, Sierra R, Sucato D, Sink E, Beaule P, Millis MB, Podeszwa D, Clohisy JC. Complications associated with the periacetabular osteotomy: a prospective multicenter study. J Bone Joint Surg Am. 2014; 96:1967-1974. 